\title{
Topology and Application Aware Dynamic VM Management in the Cloud
}

\author{
Michael Tighe $\cdot$ Michael Bauer
}

Received: 25 May 2016 / Accepted: 21 March 2017 / Published online: 2 May 2017

(C) The Author(s) 2017. This article is an open access publication

\begin{abstract}
Cloud computing continues to mature and more applications continue to be deployed in public clouds. Client applications deployed in the cloud should automatically scale up and down to match changing workload demands, though they must be careful to ensure that sufficient resources are provisioned to achieve performance objectives. The cloud provider, on the other hand, attempts to reduce costs by reducing power consumption by consolidating load onto fewer, highly utilized machines. In this work, we introduce an algorithm that integrates both application autoscaling and dynamic virtual machine (VM) allocation into a single algorithm in order to achieve the goals of both cloud provider and client. Further, we consider multi-VM applications, such as multitiered web-based applications, and extend the integrated algorithm to take the network topology into account when placing or migrating applications. The goal is to reduce VM-to-VM communication latency; our focus is on trying to contain applications within
\end{abstract}

Support for this research was provided by the Natural Sciences and Engineering Research Council of Canada (NSERC).

M. Bauer $(\bowtie) \cdot$ M. Tighe

Department of Computer Science, The University

of Western Ontario, London, Ontario, Canada

e-mail: bauer@csd.uwo.ca

M. Tighe

e-mail: mtighe2@csd.uwo.ca the same racks. We evaluate our work through simulation, showing that the integrated algorithm can achieve better application performance with a significant reduction in virtual machine live migrations, and the topology-aware extension successfully places applications within a single rack.

Keywords Data center management .

Virtualization · Cloud management · Energy management $\cdot$ SLA management $\cdot$ Application scaling $\cdot$ Autoscaling

\section{Introduction}

As the popularity of cloud computing continues to skyrocket, applications are increasingly being deployed in public clouds. The cloud, at least in theory, provides application owners with access to a seemingly infinite pool of resources, which they can acquire and release whenever needed. In the process, they save money, reduce their infrastructure management responsibilities, and enable their application with the ability to quickly scale to meet demand. Public cloud offerings are typically divided into three, very different, types: Infrastructure as a Service (IaaS), Platform as a Service (PaaS), or Software as a Service (SaaS). This work focuses on a public, virtualized IaaS environment, providing resources in the form of virtual machines (VMs) with low-level access to clients. Virtualization technology allows a physical 
machine (which we refer to as a host) to run multiple virtual machines, each with its own portion of the total physical resources, and each operating as if it were a physical machine with no knowledge of the other VMs co-located with it. Clients provision VMs of various specifications on demand, and are charged in a pay-per-use manner. The cloud provider must place these VMs in the data center in such a way that their performance goals are met and power consumption is minimized, considering resource allocations on hosts as well as the topology of the data center. There are three main actors to consider in this situation: the provider, the client, and the user. The cloud provider is the owner of the cloud infrastructure, which is being rented to the clients. Clients provision VMs from the provider in order to deploy their applications in the cloud, which provide services to users. The provider serve its clients, and the clients serves their users.

One of the key advantages to moving an application into the cloud is the ability to dynamically scale the application to meet changes in demand. This essentially gives the application owner an elastic infrastructure, scaling to meet increasing demand and scaling down when demand is low and resources are underutilized. This process, known as autoscaling, can be accomplished by adding or removing VMs to and from the application. For example, typical web applications experience highly variable loads, with average demands coming in far below peak. If enough resources are provisioned to meet the peak load (and likely more, since it may not be possible to accurately predict peak resource requirements), then a large amount of the provisioned resources will be significantly underutilized. This results in increased costs as the client is charged based on resources provisioned, not by actual utilization. Conversely, if the application is provisioned for average load, then performance can suffer during peak periods, causing potential loss of customers and business. Therefore, in order to both reduce costs while still meeting performance requirements, applications must dynamically add and remove resources, in the form of virtual machines, to match ever-changing demands from users. Commercial implementations of autoscaling exist, such as Amazon Web Services' Auto Scale [1], which allow autoscaling rules to be defined by the client.

In addition to simply providing virtual machines to clients, the IaaS cloud provider faces a set of challenges to make the best use of their infrastructure. A typical server consumes $50 \%$ to $70 \%$ of its peak power even when completely idle [4], and as such, underutilized servers represent a significant waste of power. In order to reduce power consumption, VMs must be consolidated onto the fewest number of hosts possible, with idle machines being switched off or into a power saving mode. The use of virtualization allows for better use of physical resources, but still falls short of achieving a high utilization. A tighter packing of VMs can be achieved by overcommitting resources, such as CPU; that is, a host can promise more CPU resources to its set of VMs than it actually possesses, betting on the assumption that not all VMs will require their maximum CPU request at once, allowing the aggregate CPU consumption to remain high but not overloaded. This approach, however, can lead to contention when VMs do, in fact, require more resources than are actually available on the host. In a public cloud environment, where predictions of dynamic VM resource requirements are unlikely to be available, such resource contention is unavoidable. A dynamic approach to resource allocation, where allocations are adapted at run-time to match current demands, is therefore required. This can be accomplished through the use of VM live migration, which moves a running VM from one host to another. Migration happens seamlessly, without significant downtime, but nevertheless incurs some network and performance overhead. Therefore, excessive migrations should be avoided. Finding an optimal placement of VMs, given their current resource requirements, is known to be an NP-hard problem [17]. Given this limitation, most work that deals with VM allocation at a large scale (as in a large public cloud) makes use of first-fit or best-fit heuristics [5, 19, 33], periodically recalculating VM allocations as the resource utilization of VMs changes over time. We refer to this process as Dynamic VM Allocation.

Autoscaling and dynamic VM allocation is further complicated by the fact that many clients do not simply deploy a single VM in the cloud, but rather a set of interacting VMs, each of which is a component of a larger application providing a service to their users, e.g., multi-tiered web-applications or scientific applications with multiple processing steps [8, 16, 38, 43]. For the purposes of this work, we define an application as a set of interacting VMs which provide a user-facing service. The cloud provider, upon request from a client, places client VMs within the physical 
data center. The placement should consider the data center network topology, as this can have an effect both on application performance as well as management. For example, VMs that communicate frequently with each other would benefit from being located near each other in order to reduce network latency. Adding an additional VM to handle additional web requests, say, should help, but placing it away from the VM providing database services for the overall application may not result in the performance expected.

While there is significant existing work examining autoscaling and dynamic VM allocation, most work looks at one or the other, in isolation. The goal of this work is to develop a unified approach to support both operations, and to leverage basic control over autoscaling to assist in dynamic VM allocation and fulfil the goals of both the cloud provider and the cloud client. The goals of the cloud client are to a) ensure service level agreements (SLA) with their users and $b$ ) provision the smallest amount of resources possible. Similarly, the goals of the cloud provider are to a) reduce infrastructure usage to conserve power, b) ensure client VMs have resources they require and c) avoid affecting application autoscaling decisions which could unfairly increase client costs. Finally, the cloud provider needs to also consider the network topology of the data center when placing applications, as well as when performing VM live migration [3, 39, 45]. Communicating VMs should be placed close to each other in the network (e.g. within the same rack) in order to both reduce latency between VMs and alleviate bandwidth consumption of higher level network links. Similarly, migrations should occur over the fewest number of network links possible.

Considering this set of goals, we present a new approach to handling SLA-aware application autoscaling and topology-aware dynamic VM allocation. The contributions of our work are twofold. First, we provide an integrated algorithm to support both autoscaling and dynamic VM allocation in such as way as to allow autoscaling decisions to remain under the control of the client, allowing for alternative rules or autoscaling algorithms customized for their specific application, without modification to the primary algorithm. Autoscaling decisions are made considering only the best interests of the application, with the execution of these decisions being handled in a manner that helps achieve dynamic VM allocation goals. This part of the paper is an extension of previously presented work [34]. Second, we modify and extend this integrated algorithm by adding topology-aware application placement. Further contributions included in this paper include: a detailed problem definition; an expanded description of the application model; enhanced evaluation, including dynamic application arrivals and departures during experiments; and additional reporting on evaluation results.

The remainder of the paper is organized as follows: Related work is introduced in Section 2. Section 3 defines the problem more precisely. We present a method for automatically scaling applications in Section 4. In Section 5, we introduce dynamic VM allocation by way of an existing algorithm for VM consolidation, relocation and placement. Section 6 presents a new algorithm integrating autoscaling and dynamic VM allocation, and Section 7 describes a topology-aware extension. Finally, we evaluate our work in Section 8, and conclude in Section 9.

\section{Related Work}

Existing related work can be considered in three categories: work on autoscaling, work on dynamic VM allocation, and work on topology-aware placement.

\subsection{Autoscaling}

There have been several extensive surveys of approaches to autoscaling or elasticity in the cloud. Singh and Inderveer [30] carry out an extensive survey of resource allocation in clouds, which includes looking at approaches to resource scheduling generally, but also specific algorithms for provisioning (VM allocation) and dynamic allocation of VMs and migration. They provide a taxonomy for classifying resource allocation algorithms in cloud environments. Based on their extensive survey, they identify a number of challenges and issues, including autonomic management for dynamic resource provisioning, detecting SLA violations, VM migration and consolidation and energy management. The integrated algorithm presented in this paper addresses these issues through an integrated approach that considers the location of dependent VMs.

Other researchers have focused specifically on approaches to autoscaling in cloud environments. Lorido-Bortran, et al. [23] considered a variety of 
autoscaling approaches focusing specifically on elastic applications, i.e., applications that can be scaled horizontally or vertically. In horizontal scaling the resource unit is the server replica running on a VM and and replicas are added or released as needed. Vertical scaling consists of changing the resources assigned to an already running VM, e.g., increasing or reducing cpu resources allocated to that VM. They grouped approaches into five categories by techniques used: threshold based rules, reinforcement learning, queuing theory, control theory and time series analysis. For example, Amazon Web Services provides an autoscale feature (AWS Auto Scale) [1], allowing clients to specify conditions, i.e., rules, under which additional VM instances should be added or removed. Naskos et al. [26] consider elasticity from the perspective of a MAPE loop [20, 25]: Monitoring, Analysis, Planning and Execution, with particular focus on the last three phases and how difference approaches handle those aspects. Galante and de-Bona [11] also provide a survey of both commercial and academic approaches to elasticity in clouds. Again, they consider approaches that focus on horizontal or vertical scaling of applications. They do, however, also identify a few approaches that consider VM migration when migration would result in a VM migrating to a host that provided needed resources. Ghanbari et al. $[12,13]$ examine alternative autoscaling approaches in the literature classifying them into two categories: control theory approaches, and rule-based heuristics.

Much of the work on autoscaling and elasticity is from the perspective of the client running applications within the cloud and so look to provide clients with means of specifying constraints for scaling, e.g. conditions or thresholds; some work does tie scaling to SLAs. For example, Ferretti et al. [9] autoscale applications based on SLA achievement. They specify a threshold on response time as their SLA objective, and attempt to proactively scale the application based on continuous monitoring. Most of the literature focuses on scaling resource allocations of a single VM to meet its current demands, but do not scale applications out to multiple VMs [29].

\subsection{Dynamic VM Allocation}

A great deal of work has been done in the area of dynamic VM allocation for the purpose of VM consolidation and thus power conservation. Early work examined statically allocating VMs such that their resource demands would not be in conflict, based on the assumption that demands exhibit a periodic cycle. Approaches using a best-fit heuristic [7], linear programming [31] and vector bin packing [33] have been proposed. Other approaches have considered variable demand by periodically recalculating the entire VM allocation [6]. Work on truly dynamic VM allocation focuses mostly on first-fit [21,38] or best-fit [5] heuristic solutions. Wood et al. [40] employ a firstfit decreasing heuristic that spreads load across hosts to reduce SLA violations. Keller et al. [19] studied several variations on a first-fit heuristic, concluding that the order in which VMs and hosts are considered for migration can have an impact on metrics such as power consumption and SLA violations. Some alternatives to best or first-fit heuristics, such as fuzzy logic-based controllers [14], have also be investigated.

There are a small number of works that examine something similar to combining autoscaling with dynamic VM allocation. Wuhib et al. [41] propose an approach to distributed load-balancing in the cloud using a gossip protocol. Hosts balance workloads between themselves by adjusting load-balancing settings as well as by starting and stopping module (application) instances. The work was extended [42] to also consolidate workload for the purposes of reducing power consumption. The proposed solutions make use of a demand profiler to estimate resource requirements of modules and targets a Platform as a Service (PaaS) cloud, although the authors claim that the approach could be adapted to manage an IaaS cloud. Our approach does not require a demand profiler. Furthermore, their application scaling is performed with the goal of load balancing, rather than reducing the costs of clients running applications in the cloud. We focus on letting applications decide how they want to scale, and providing them with the resources they require.

Jung et al. [18] perform application scaling and dynamic VM allocation using a layered queueing model of applications to determine the utility of configurations. They also require offline experimentation to determine the effects of configuration adaptation methods, which are later used to estimate effects online. Our approach does not require detailed models nor prior offline processing. Petrucci et al. [27] use a mixed integer programming model to optimize application placements. Unlike our work, they do not allow 
the client to determine auto-scaling conditions and the scalability of their approach is not fully evaluated.

\subsection{Topology-Aware Placement}

Some previous work has taken topology of the cloud system or data center into consideration when initially placing VMs. Gupta et al. [16] examine improving the performance of HPC applications in the cloud through intelligent VM placement. Their approach is topology-aware, with the goal of placing VMs on physical machines as close as possible to each other in order to provide greater bandwidth and reduce latency between nodes in the application. Yapicioglu and Oktug [43] place VMs into hosts in order to minimize inter-rack traffic, reducing communication delay and saving power costs in networking elements. Dias and Costa [8] also attempt to cluster related VMs near each other to reduce network congestion and core network traffic.

Other work considers the data centre topology when placing applications. SecondNet [15] handles placement of virtual data centres (VDCs). VDCs not only define a set of VMs, but also the communication links required between them. SecondNet is capable of providing bandwidth guarantees between VM pairs, and of modifying the VDC allocation in response to scaling or server failures. They also perform live migration in order to move VMs in a VDC closer together (fewer network hops), when possible. Zhani et al. [44] introduce VDCPlanner, which also focuses on VDC placement, as well as scaling and dynamic consolidation. Finally, Mann et al. [24] present Remedy, a VM management system which takes data centre networking into consideration. Remedy monitors bandwidth utilization, and makes use of VM migrations to shift load and relieve congested network links. In VDCPlanner [44], Zhani et al. attempt to minimize the communication distance between VMs in a virtual data centre in order to reduce bandwidth consumption and allow unused networking elements to be turned off. Our work differs from existing topology-aware application placement in that we consider data centre topology while performing dynamic VM requirements and allocation with overcommitting of resources. Ballani et al. [2] propose allowing cloud and datacentre clients to specify a virtual cluster topology, in order to provide improved and more stable application performance.
They claim that a significant amount of variability in cloud application performance can be attributed to variable network bandwidth between communicating VMs. Unlike our work, they do not consider dynamic VM allocation/placement.

The placement of virtual machines to minimize virtual machine or resource interference has also been considered in some work [38, 45]. This work has typically focused on the use of VMs to support high performance or scientific applications where there are multiple dependent servers/tasks that interact.

We focus on combining autoscaling and topologyaware dynamic VM allocation into a single algorithm, capable of scaling applications while also consolidating load for power conservation and network load. Other work considers only one of these goals, or targets a different environment.

\section{Problem Definition}

In this section, we present the problem that we are addressing. We begin with a description of the dynamic VM allocation problem, followed by a description of the model we are using for applications running in the cloud. Finally, we look at some considerations with regards to application placement within the data centre network topology, as well as management operations. Table 1 provides a summary of the notation used in the description of the problem and the algorithms.

Figure 1 provides a general overview of the structure of hosts $\left(h_{1}, h_{2}, \ldots\right)$, racks $\left(r_{1}, r_{2}, \ldots\right)$, VMs, and applications. An application, $a_{i} \in A$, contains a set of tasks, $T_{a}$, where a single task is denoted $t_{j} \in T_{a}$. Tasks represent different components of the application, such as a web server(s), application server(s), or database. Tasks are performed by one or more identical task instances, $i_{k} \in I_{t}$, where $I_{t}$ is the set of task instances in task $t$. Task instances are the actual deployed software components that perform the task. A VM, $v_{i_{k}}$, is assigned to run a single task instance $i_{k}$.

\subsection{Dynamic VM Allocation}

At the most basic level, the problem of dynamic VM allocation is to place a set of VMs, $V$, into a set of hosts, $H$, and to dynamically adapt this placement as the resource demands of VMs change. Hosts are 
Table 1 Summary of Notation

\begin{tabular}{|c|c|}
\hline Symbol & Definition \\
\hline$A ; a, a_{i}$ & Set of applications; applications \\
\hline$H ; h, h_{j}$ & Set of hosts; hosts \\
\hline$T_{a} ; t, t_{k}$ & Set of tasks; tasks \\
\hline$I_{t} ; i, i_{l}$ & Set of task instances; instances \\
\hline$\left|I_{t}\right|$ & Number of task instances for a given task \\
\hline$\left|I_{t}\right|^{\max }$ & $\begin{array}{l}\text { Maximum number of task instances for a } \\
\text { given task }\end{array}$ \\
\hline$V, V_{t}$ & Set of VMs \\
\hline$V_{i_{l}}$ & VM running instance $i_{l}$ \\
\hline$R ; r, r_{m}$ & Set of racks; racks \\
\hline$H_{r}$ & Set of hosts in rack $r$ \\
\hline$s$ & Simulation time \\
\hline$\Omega$ & Host cpu \\
\hline$\omega$ & VM cpu \\
\hline$T^{\uparrow} ; T^{\downarrow}$ & Tasks to scale up; down \\
\hline$T_{a}$ & Tasks for application $a$ \\
\hline$\rho ; \rho^{\tau}$ & Response time; threshold (SLA) \\
\hline$W_{\rho}$ & $\begin{array}{l}\text { Size of sliding window for average } \\
\text { response time }\end{array}$ \\
\hline$W_{\omega}$ & $\begin{array}{l}\text { Size of sliding window for average cpu } \\
\text { utilization }\end{array}$ \\
\hline$S^{\text {warn }}$ & SLA warning value (\% of $\rho^{\tau}$ ) \\
\hline$\omega^{\text {warn }}$ & Warning threshold for cpu utilization \\
\hline$\omega^{\text {safe }}$ & Lower threshold for utilization of cpu \\
\hline$H^{!}$ & Stressed hosts (utilization greater than $\bar{\Omega}^{\tau}$ ) \\
\hline$H^{+}$ & $\begin{array}{l}\text { Partially stressed hosts (utilization } \\
\text { between } \underline{\Omega}^{\tau} \text { and } \bar{\Omega}^{\tau} \text { ) }\end{array}$ \\
\hline$H^{-}$ & Under utilized hosts (utilization below $\underline{\Omega}^{\tau}$ \\
\hline$H^{\emptyset}$ & Hosts not hosting any VMs \\
\hline$\Theta^{\tau}$ & $\begin{array}{l}\text { Limit on the number of times a host can } \\
\text { be stressed }\end{array}$ \\
\hline$\theta_{h}$ & Number of times host $h$ has been stressed \\
\hline$\Omega^{\Gamma}$ & Estimated reduction in cpu utilization \\
\hline$\Omega^{\Delta}$ & $\begin{array}{l}\text { Threshold for cpu utilization for } \\
\text { determining stressed hosts }\end{array}$ \\
\hline$\Upsilon_{h}$ & Target number of active hosts in a rack \\
\hline$\omega_{v}(s)$ & cpu utilization for VM $v$ at time $s$ \\
\hline$\omega_{v}^{\max }$ & Upper limit for cpu utilization for VM $v$ \\
\hline$\alpha_{h, v}(s)$ & True if host $h$ is on VM $v$ at time $s$ \\
\hline$M_{h}$ & Memory capacity of host $h$ \\
\hline$\Omega_{h}$ & Maximum cpu usage of host $h$ \\
\hline$\Omega_{h}^{\prime}(s)$ & cpu usage of host $h$ at time $s$ \\
\hline$\tau$ & Upper threshold on cpu utilization of host $h$ \\
\hline $\bar{\tau}_{h}(s)$ & $\begin{array}{l}\text { Amount of cpu utilization of host h that } \\
\text { exceeds threshold } \tau \text { at time } s\end{array}$ \\
\hline$\rho_{a}(s)$ & Response time of an application $a$ at time $s$ \\
\hline
\end{tabular}

Table 1 (continued)

\begin{tabular}{ll}
\hline Symbol & Definition \\
\hline$\rho_{a}^{\tau}$ & $\begin{array}{l}\text { SLA upper threshold on response time for } \\
\text { application } a\end{array}$ \\
$\overline{\rho_{a}\left(W_{\rho}\right)}$ & $\begin{array}{l}\text { Average response time of application } a \\
\text { over window } W_{\rho}\end{array}$ \\
$\rho_{a}^{\Delta}$ & $\begin{array}{l}\text { Change in response time between windows } \\
\rho_{t}^{\text {task }}(s)\end{array}$ \\
$\overline{S_{a}}\left(s_{i}, s_{j}\right)$ & $\begin{array}{l}\text { Function which returns the response time } \\
\text { of a task at time } s\end{array}$ \\
$\overline{S_{a}^{v}}\left(s_{i}, s_{j}\right)$ & $\begin{array}{l}\text { \% time application } a \text { achieved SLA over } \\
\text { time interval }\left[s_{i}, s_{j}\right]\end{array}$ \\
$\alpha^{\Delta}(s)$ & $\begin{array}{l}\text { time interval }\left[s_{i}, s_{j}\right] \\
\text { Number of migrations that occur between }\end{array}$ \\
$\overline{\omega(i, \text { window })}$ & $\begin{array}{l}\text { time } s-1 \text { and } s \\
\text { Average CPU utilization of a task instance }\end{array}$ \\
\hline
\end{tabular}

located within racks, which are sets of hosts directly connected via a single switch. Racks are connected to each other through additional layers of networking, the details of which are not considered for the purpose of this work. We denote a rack as $r \in R$, and the set of hosts in $r$ as $H_{r} \subseteq H$.

We consider two characteristics of a VM $(v \in V)$ :

- Its CPU usage: denoted $\omega_{v}(s)$. It also has a fixed upper limit on CPU usage, $\omega_{v}^{\max }$. The CPU utilization of a VM is therefore $\omega_{v}(s) / \omega_{v}^{\max }$. CPU usage is quantified in terms of $C P U$ units, in which a single unit is equivalent to $1 \mathrm{MHz}$ clock speed. For example, a $2.5 \mathrm{GHz}$ processor core would have $2500 \mathrm{CPU}$ units.

- Its memory: denoted $\mu_{v}$, memory size is static.

The placement (also called allocation) of a VM, is defined by the following function:

$\alpha_{h, v}(s)= \begin{cases}1 & \text { if } v \text { is placed on } h \\ 0 & \text { otherwise. }\end{cases}$

A VM can only be placed on a single host at a time. Therefore, if there exists a host $h_{1}$ such that $\alpha_{h_{1}, v}(s)=$ 1, then $\forall h \in H \backslash h_{1}, \quad \alpha_{h, v}(s)=0$.

Each host, $h \in H$, has a total memory capacity $M_{h}$. The placement must be such that the sum of the memory allocations for each VM on a host does not exceed the memory capacity of the host.

$\sum_{v \in V} \mu_{v} * \alpha_{h, v}(s) \leqslant M_{h}, \quad \forall h \in H \quad \forall s \in \mathbb{R}^{+}$. 
Fig. 1 Data centre model

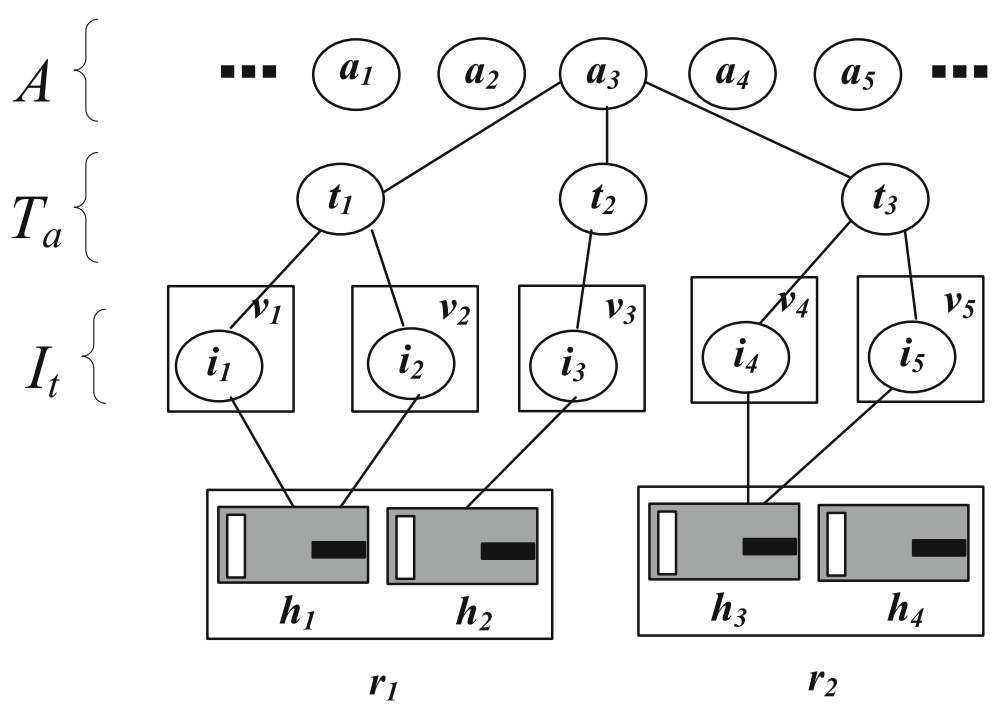

Each host, $h$, has a total CPU capacity $\Omega_{h}$. The CPU usage of a host, $\Omega_{h}^{\prime}(s)$, is equal to the sum of the CPU usage of all VMs placed on that host, and cannot exceed the total CPU capacity of the host. Consequently, for $s \in \mathbb{R}^{+}$, we have:

$\Omega_{h}^{\prime}(s)=\sum_{v \in V} \omega_{v}(s) * \alpha_{h, v}(s) \leqslant \Omega_{h}$.

Note that this is not a rule that must be enforced, but rather is a physical property of the host.

For simplification, we denote the set of VMs on a host $h$ as $V_{h}$, Consequently, we have: $v \in V_{h} \Longleftrightarrow$ $\alpha_{h, v}=1$.

It is important to avoid overloading the CPU of a host, in order to avoid compromising the performance of VMs placed on it. To this end, we define an upper threshold on CPU utilization, $\tau$ (e.g. 0.9, or 90\%). We calculate the amount by which each host, $h$, exceeds $\tau$. We denote this as $\bar{\tau}_{h}(s)$, and calculate it as follows:

$\bar{\tau}_{h}(s)= \begin{cases}\frac{\Omega_{h}^{\prime}(s)}{\Omega_{h}}-\tau & \text { if } \frac{\Omega_{h}^{\prime}(s)}{\Omega_{h}}-\tau>0 . \\ 0 & \text { otherwise. }\end{cases}$

Then, our goal is to minimize this value for all hosts, which gives use the following objective:

$\forall s \in \mathbb{R}^{+}, \min \sum_{h \in H} \bar{\tau}_{h}(s)$.

A second goal is to reduce power consumption, which is accomplished by minimizing the number of active hosts. An active host contains at least one VM. The number of VMs on a host is denoted $|h|$. Formally, a host is active at time $s \in \mathbb{R}^{+}$if the following function is equal to 1 :

$\operatorname{active}(h, s)= \begin{cases}1 & \text { if }|h|(s)>0 . \\ 0 & \text { if }|h|(s)=0 .\end{cases}$

By minimizing the number of active hosts, we can ensure that hosts are not underutilized. Since an idle server can still use $50 \%$ to $70 \%$ of their maximum power consumption, higher host utilization results in better power efficiency in terms of CPU processing per watt. Therefore, the total number of active hosts should be minimized, giving us the following objective:

$\forall s \in \mathbb{R}^{+}, \min \sum_{h \in H} \operatorname{active}(h, s)$.

\subsection{Applications}

Every VM within the data centre is running a component of an application. In this work, we consider interactive applications, such as a multi-tiered web application, in which a number of clients interact with the system, waiting for responses before issuing a new request. There is a single class of requests, entering and exiting the application through a single point.

An application, $a \in A$, contains a set of tasks, $T_{a}$, where a single task is denoted $t \in T_{a}$. Tasks represent different components of the application, such as a web server(s), application server(s), or database. Tasks are performed by one or more identical task instances, 
$i \in I_{t}$, where $I_{t}$ is the set of task instances in task $t$. Task instances are the actual deployed software components that perform the task. For example, a web server task of an application may consist of a deployed set of replica web servers, with incoming requests load balanced between them. A VM, $v_{i}$, is assigned to run a single task instance $i$. Furthermore, $\forall i \exists ! v_{i}$. That is, every task instance must be running within a single VM.

For any application, at least one instance of each task in the application must exist. That is, $\left|I_{t}\right|>$ $0, \quad \forall t \in T_{a}$. The number of instances in each task can change over time, as instances are added or removed dynamically to match current workload demands. Task instances share the total workload (i.e. requests) of their tasks, and in this work, it is assumed that the workload is distributed equally.

We consider the Service Level Agreement (SLA) for an application to be defined as an upper threshold on application response time. The response time of an application $a \in A$, at time $s$, is denoted $\rho_{a}(s)$. Similarly, its SLA threshold is denoted as $\rho_{a}^{\tau}$. If the response time of an application falls below its threshold, then the SLA is said to be achieved. Otherwise, SLA is violated. The SLA achievement of an application is:

$\forall s \in \mathbb{R}^{+}, \quad S_{a}(s)= \begin{cases}1 & \text { if } \rho_{a}(s) \leqslant \rho_{a}^{\tau} . \\ 0 & \text { otherwise. }\end{cases}$

Conversely, the SLA violation is defined by:

$S_{a}^{v}(s)=1-S_{a}(s)$.

The percentage of time for which SLA is achieved over a time interval $\left[s_{i} . . s_{j}\right]$ is defined as:

$\overline{S_{a}}\left(s_{i}, s_{j}\right)=\frac{\sum_{s_{i}}^{s_{j}} S_{a}(s)}{s_{j}-s_{i}}$.

Similarly, the percentage of time for which SLA is violated over a time interval $\left[s_{i} . . s_{j}\right]$ is defined as:

$\overline{S_{a}^{v}}\left(s_{i}, s_{j}\right)=1-\overline{S_{a}}\left(s_{i}, s_{j}\right)$.

For simplification, we refer to the overall SLA achievement of an application over the entire time interval under consideration (e.g. the duration of an experiment) simply as $\overline{S_{a}}$. Our third goal consists of minimizing SLA violation:

$\min \sum_{a \in A} \overline{S_{a}^{v}}$

\subsection{Application Placement}

Tasks in an application must communicate with each other in order to process user requests. We assume that, for the purpose of reducing network latency, it is desirable to place all task instances of an application within a single rack, when possible. Furthermore, this reduces network usage of higher level switches (i.e. above rack-level). We define the spread of an application, $\operatorname{spread}_{a}(s)$, as the number of racks which contain VMs running task instances of application $a \in A$.

$\min \sum_{a \in A} \operatorname{spread}_{a}(s)$.

There are situations in which it may be desirable to place application components in physically distinct locations, e.g., for fault tolerance purposes. This can mean placing replicated components in different clusters of a data centre, or more likely, in different data centre locations entirely. For the purposes of this work, we do not consider this situation. We believe that this simplification is reasonable, as including such placement considerations would represent an extension of this work, rather than an invalidation of it.

\subsection{VM Live Migration}

In order to modify the placement of VMs within the data centre, a live migration operation is performed. This operation consists of transferring the state of a running VM to another host, and incurs both performance and network overhead. Therefore, an additional goal is to minimize the number of migrations required to perform dynamic VM management. The number of migrations that occur between time $s-1$ and $s$ is:

$\alpha^{\Delta}(s)=\frac{\sum_{h \in H, v \in V} \alpha_{h, v}(s-1) \oplus \alpha_{h, v}(s)}{2}$.

Note that since every placement change for a VM results in the modification of $\alpha_{h, v}$ for both hosts 
involved in the move, we divide the sum of the placement changes by two.

Our goal is to minimize the number of migrations that are performed overall, rather than at a specific point in time. Thus, we are interested in strategies that may be somewhat more conservative in when to migrate. For example, overloading a single VM that is part of a group of VMs comprising an application may not be a judicious choice to migrate, as it might move that VM further from the other VMs that it must communicate with. Rather, a strategy that considers the overall application response time and looks to migrate all the VMs of that application to a single rack would be our preferred choice since that would reduce communication latency and enhance response time. Therefore, we introduce the following objective which aims to minimize the overall number of migrations:

$\forall s \in \mathbb{R}^{+}, \quad \min \sum_{s_{1}}^{s_{n}} \alpha^{\Delta}(s)$.

\section{Application Autoscaling}

In this section, we present the application model used in this work, as well as a basic autoscaling algorithm.

\subsection{Application Model}

Applications are typically deployed with enough spare resource to handle peak traffic and workload demands. As a result, they spend most of their time significantly overprovisioned, resulting in an underutilization of resources and increased costs [9]. By dynamically scaling applications to match changing workload demands, the client can use only the resources it requires, releasing unused resources to save costs. In this work, we examine autoscaling resources through the addition or removal of VMs (task instances) to and from the application. We do not look at scaling resource allocations on hosts to individual VMs. We present a rule-based, heuristic autoscaling algorithm for use in our implementation. It would be possible, however, to make use of a more complex autoscaling algorithm without modification to our final, application-aware dynamic allocation algorithm (Section 6).
As described in Section 3, we consider an interactive application in which a number of clients interact with the application, waiting for responses before issuing a new request. We implement a model of such an application in an open source simulator, DCSim $[35,36]$, for the purposes of evaluating our approach. Figure 2 shows the structure of a basic application, that might be deployed in our evaluation, consisting of two tiers. Each tier has a load balancer component which evenly divides incoming requests between all task instances in the tier. Both tiers are able to dynamically add or remove instances (autoscale) to match current workload demands. The size of a task refers to the number of task instances it contains.

Within the simulation, we model the application as a closed queueing network, and solve it using Mean Value Analysis (MVA) [28]. We make use of some additional information about the applications and tasks. An application has a specified think time for clients, which defines the amount of time that clients wait in between receiving a reply from their previous request and sending another. It also contains a workload component, which defines the current number of clients using the application. The workload changes at discrete time points in the simulation, based on a trace file. The description of a task is expanded to include:

- default and maximum size, which defines the default and maximum number of task instances;

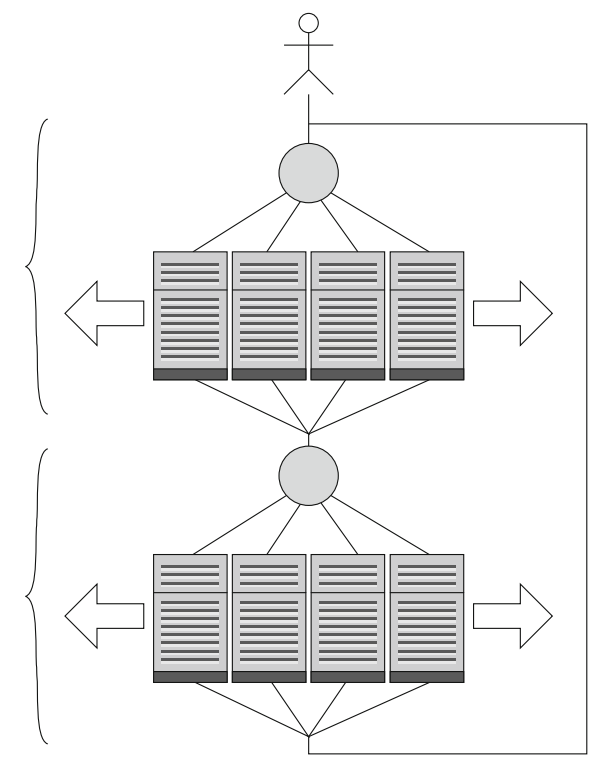

Fig. 2 Application example 
- service time, which defines the time it takes to process a single request;

- visit ratio, which defines the average number of times each request must visit the task;

- and resource size, which defines the expected amount of CPU and memory allocated to each task instance of the task, as well as the expected CPU speed, in order to achieve the specified service time.

The actual service time for each task instance may be scaled based on a CPU speed factor. The service time may also be adjusted in the case of CPU contention or overload which could be due to process queuing due to too many processes, delays due to excessive swapping, etc. We add an additional delay to the service to account for these overload situations.

The performance of a deployed application is evaluated based on its SLA achievement (see Section 3). Note that the SLA is based on the response time seen by the application users, and defines an agreement between cloud clients and their users, rather than the cloud provider and client. A lower SLA achievement (higher application response time), indicates that the application is underprovisioned, either due to a failure of the autoscaling algorithm to scale up the application, or by contention with other VMs caused by poor VM placement and dynamic management. By comparing experimental results running only the autoscaling algorithm with those running both autoscaling and dynamic VM management, we can then determine how much SLA violation is attributable to the cloud provider.

\subsection{Algorithm}

We have implemented a basic autoscaling algorithm, which scales applications up and down based on current SLA achievement and CPU utilization. We make use of a rule-based, heuristic algorithm, as it is lightweight and does not require detailed knowledge or models of the applications being managed. Furthermore, with such an algorithm, it would be possible for application owners (cloud clients) to define their own threshold values and rules for autoscaling, customized to their own needs and goals.

We assume that each application has a single manager, which monitors the application and that task instances regularly send monitoring data to the manager. We also assume, for purposes of the simulation, that the autoscaling algorithm (Algorithm 1) executes on a fixed interval (e.g. every 5 minutes). Of course, in practice these need not be the case; this could be an area for future study where alternative decision making algorithms are used to determine when the autoscaling algorithm should be executed. The algorithm requires the following as input:

- $\quad$ the application being scaled $(a)$;

- the time $(s)$;

- the window sizes for sliding average response time $\left(W_{\rho}\right)$ and CPU utilization $\left(W_{\omega}\right)$;

- a Response Time Threshold $\left(\rho^{\tau}\right)$ defining the SLA of the application;

- an SLA Warning value $\left(S^{\text {warn }}\right)$, specified as a percentage of the SLA response time threshold;

- $\quad$ and a CPU Safe value ( $\left.\omega^{\text {safe }}\right)$.

We calculate the average response time, $\rho_{a}$, over a window $W_{\rho}$ using the function $\overline{\rho_{a}\left(W_{\rho}\right)}$ (line 1$)$. Then, $\rho_{a}$ is compared against the warning threshold (line 2). If it exceeds this value, a scale up operation is performed to add additional VMs to the application. The algorithm then iterates through the tasks that comprise the application (lines 4-7), and calculates the change in response time $\left(\rho_{a}^{\Delta}\right)$ since the last execution of the algorithm. We assume a function $\rho_{t}^{\text {task }}(s)$, which returns the response time of a task at a specified time. The algorithm chooses the task with the largest increase in response time, and whose size, $\left|I_{t}\right|$, has not reached its maximum size, $\left|I_{t}\right|^{\text {max }}$. A new instance is then added to this task (line 8).

If the response time is below the warning threshold, the algorithm looks for a task on which to perform a scale down operation. Scaling down is performed based on CPU utilization, rather than of the SLA. The function $\overline{\omega(i, \text { window })}$ computes the average CPU utilization of a task instance over a sliding window. The total CPU utilization of all instances in the task $\left(\operatorname{tas}_{\theta}\right)$, averaged over a sliding window of size $W_{\omega}$ (line 12), is computed and divided by task size minus one to estimate the utilization of remaining task instances, should one be removed (line 13). If this value falls below a specified $C P U$ Safe value $\left(\omega^{\text {safe }}\right)$, then the task is a candidate for a scale down operation. The algorithm chooses the candidate task with the lowest utilization from which to remove an instance 
(lines 14-15), and removes an arbitrary instance from the task (line 16).

The threshold values for SLA Warning and CPU Safe, as well as the metric to evaluate (e.g. response time, throughput, CPU utilization) could easily be defined by the application owner, in a similar manner as the rules defined in Amazon Web Services Auto Scale [1]. This gives the client control over autoscaling decisions and trade-offs between SLA performance and cost.

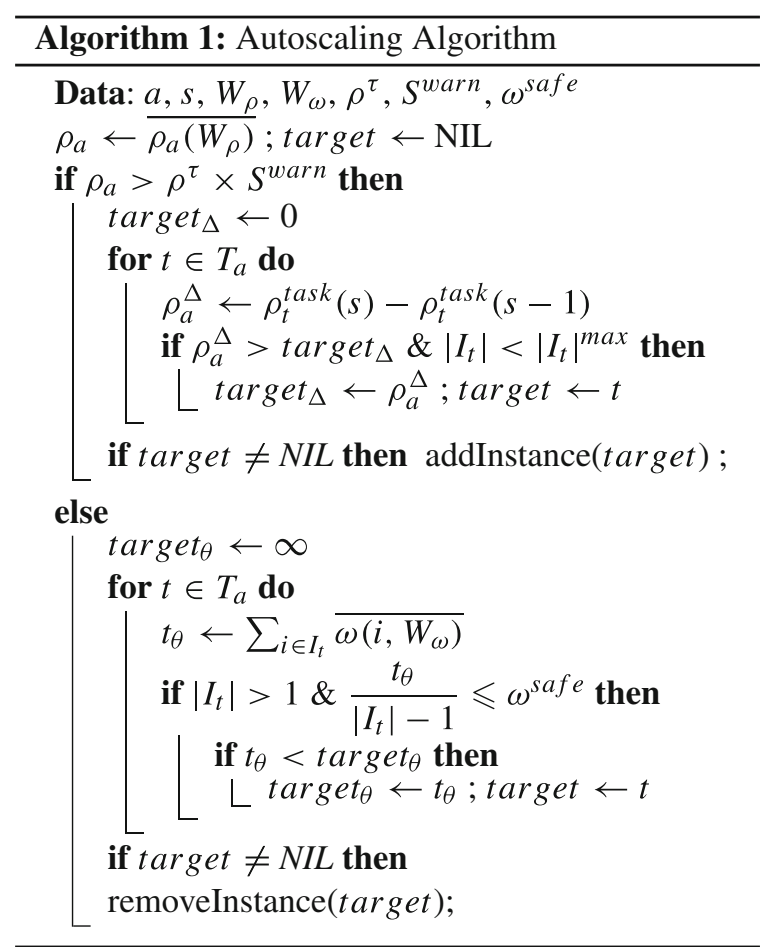

\section{Dynamic VM Allocation}

By consolidating VMs on as few hosts as possible, the cloud provider can maximize the use of their infrastructure and/or minimize power costs. This must be done, however, while still providing enough resources to VMs for them to meet their performance objectives. A tight packing of VMs on hosts can be accomplished by overcommitting resources, such as CPU; that is, by allocating more than the host's actual capacity to its VMs. As workload demand for VMs changes over time, they must be dynamically relocated to other hosts in order to ensure that enough resources are available to meet their current demand. Therefore, when a host is running low on resources (e.g. CPU), we move a VM to another host through the a live migration operation.

We use a modified first-fit heuristic algorithm as the basis of dynamic VM allocation in this work. Originally presented in our previous work [19], it is representative of a large amount of the related work in the area. Hosts are first classified into four categories:

- $\quad$ stressed hosts $\left(H^{!}\right)$have a CPU utilization higher than a specified $\bar{\Omega}^{\tau}$ threshold value;

- partially utilized hosts $\left(H^{+}\right)$have a CPU utilization below $\bar{\Omega}^{\tau}$ and above a lower threshold, $\underline{\Omega}^{\tau}$;

- underutilized hosts $\left(H^{-}\right)$have a CPU utilization below $\underline{\Omega}^{\tau}$;

- and empty hosts $\left(H^{\emptyset}\right)$ are not hosting any VMs, and may be in a low power state such as suspended or off.

Each category is sorted by CPU utilization in either ascending or descending order to control the behaviour of the algorithm. We refer the reader to previous work [10, 19, 37] for more details.

The approach consists of three basic operations, described in the remainder of this section.

\subsection{VM Relocation}

A host is considered stressed when it's CPU utilization exceeds $\bar{\Omega}^{\tau}$. At this point, the stress situation must be resolved by relocating (migrating) one of its VMs to another host. This process is known as VM Relocation, and is triggered on a regular interval (every 10 minutes in the simulation; this was based on some previous work on VM relocation strategies [10]). Algorithm 2 provides an overview of the relocation algorithm. It takes the set of hosts in the data centre $(H)$, and first classifies them as described above (line 1). The set of stressed hosts are used as migration sources (line 2), and the remaining, sorted categories are concatenated and used as the target list (line 3). The VMs of each source host $\left(V_{h}\right)$ are sorted and iterated though, searching for a target host $\left(h_{t}\right)$ for each $v \in V_{h}$ (lines 4-12). Once a suitable VM and target has been found, the algorithm performs the 
migration (line 9) and moves on to the next source host.

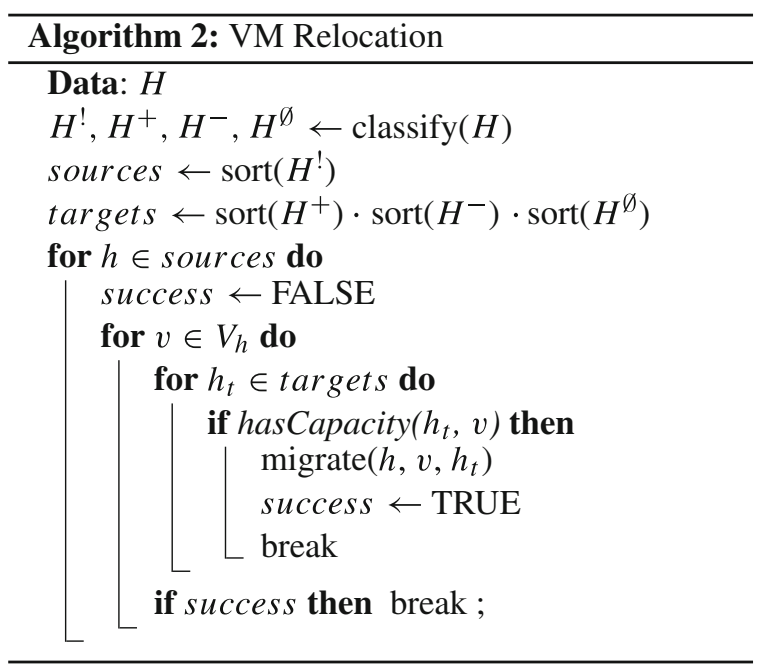

\subsection{VM Consolidation}

While the VM Relocation operation spreads out VMs in order to relieve stressed hosts, the VM Consolidation operation takes on the opposite goal by attempting to consolidate VMs on as few hosts as possible. This operation is triggered on a longer time interval than VM Relocation (every hour) [10]. When a host becomes underutilized (its CPU utilization is less than $\underline{\Omega}^{\tau}$ ), it is a candidate for having its remaining VMs evicted and being shut down (or suspended) to reduce power consumption. In order to accomplish this, target hosts are found to migrate each VM off of the candidate host, the migrations are performed, and the host is switched into a low power state (off or suspended). The algorithm is similar to the VM Relocation algorithm (Algorithm 2), except that underutilized hosts $\left(H^{-}\right)$are used as sources, and any non-stressed host can be used as a target.

\subsection{VM Placement}

The VM Placement operation is performed each time a new request to create a VM arrives in the data centre. This operation is responsible for selecting an appropriate host for the new VM, and instantiating it. Once again, it works similarly to the VM Relocation algorithm (Algorithm 2), except it selects a target host for a new VM, rather than for VMs on stressed hosts.

\section{Integrated Application-aware Algorithm}

Dynamic VM allocation requires the use of VM live migration operations in order to adapt the allocation to changing workload conditions. This operation, however, is not free. Rather, it degrades VM performance during migration, and consumes network bandwidth in order to transfer the running state of the VM. We propose a single algorithm, integrating autoscaling (see Section 4) with dynamic VM allocation (see Section 5), in order to leverage autoscaling operations to assist dynamic VM allocation and reduce the number of migrations required. In this approach, each application retains control over deciding to perform scale up and scale down operations. The choice of which task instances to shut down (in the case of a scale down operation), and where to place new instances (in the case of a scale up operation), however, are performed in such a way as to aid VM Relocation and VM Consolidation and reduce the need for live migrations.

The integrated, application-aware algorithm combines autoscaling operations with VM Relocation and $V M$ Consolidation into a single algorithm, referred to as the Dynamic Allocation operation, which is run on a regular time interval. The VM Placement operation is handled separately, triggered by the arrival of new applications to the data centre. Algorithm 3 presents a segment of the integrated algorithm, which deals with relieving stressed hosts (i.e. VM Relocation). This is the first piece of the algorithm, the remainder of which continues on to perform remaining scaling and consolidation operations. The inputs of the algorithm are the set of hosts in the data centre $(H)$, the set of running applications $(A)$, and a timeout limit on host stress $\left(\Theta^{\tau}\right)$. The algorithm attempts to resolve stress situations through careful execution of autoscaling operations. Once a host has been stressed for the specified number of algorithm executions, however, the algorithm triggers a relocation via migration rather than continuing to wait for autoscaling operations to clear the stress situation.

First, hosts are classified as described in Section 5. The number of consecutive times that the host has been stressed is then recorded in lines 2-3. In line 4 , the evaluateScaling() method executes a modified version of the autoscaling algorithm (Algorithm 1) for each application. It varies from Algorithm 1 in 
that rather than performing the autoscaling operations directly, it returns a list of tasks to be scaled up $\left(T^{\uparrow}\right)$ and scaled down $\left(T^{\downarrow}\right)$. Note that this algorithm could easily be exchanged for a more complex autoscaling algorithm, or even a different algorithm for each application, as required by the application owner. It is simply required to return the list of tasks to scale. For our current implementation, however, the algorithm presented in Section 4 is used.

The algorithm then attempts to relieve the stress situation of each stressed host, iterating through them in line 5. Line 7 calculates the amount by which CPU utilization on the host exceeds the stress threshold $\left(\Omega^{\Delta}\right)$. The first step in relieving this stress is to determine whether or not the effect of pending scale up operations will relieve the stress without further action required. Since task instances of each task share incoming work, a scale up operation will result in a portion of the work from each existing task instance being taken by the new instance. We calculate the total estimated reduction in CPU utilization for the host $\left(\Omega^{\Gamma}\right)$. The function estimateReduction $(i)$ computes the estimated reduction in CPU utilization for instance $i$. It computes this estimate based on the expected reduction in CPU utilization on the host if the instance is moved. We can do this since since we assume that each application task instance runs in a single VM and we track VM CPU utilization (see Section 5). Thus, we can estimate for each task instance on a host $h$ whether moving it would reduce CPU utilization enough to return the host to a non-stressed state (lines $8-12$ ). If the pending scale up operation(s) will sufficiently reduce the CPU utilization, then no further action is required.

Next, the algorithm looks for pending scale down operations in which the task being scaled down contains an instance which is running on the stressed host (lines 13-21). If a task is found, we select for shut down the task instance running on the stressed host (line 18). The task is then removed from the set of tasks pending scale down operations (line 19). No further action is taken. If, however, neither of the previous attempts were successful, and the host has remained stressed for the specified timeout period $\left(\Theta^{\tau}\right)$, we perform a relocation via live migration. Based on an evaluation of multiple possible values, we set the timeout value to 2 algorithm executions. Relocation is then performed in a similar manner to Algorithm 2.

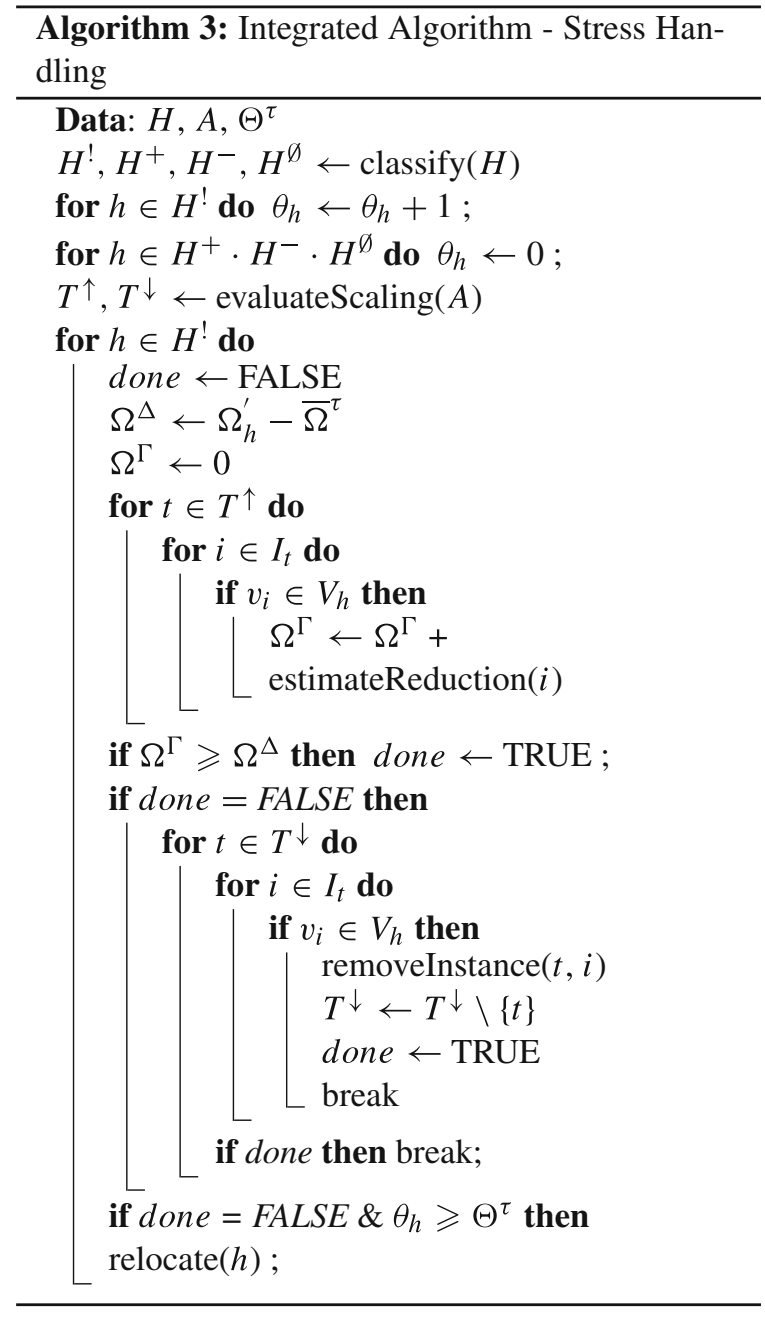

The remainder of the algorithm continues to make use of pending scale up and scale down operations to avoid migrations. First, we look for scale down operations which can help prevent hosts that are close to becoming stressed from reaching the stressed threshold. Scale down operations on tasks that have an instance located on a host with CPU utilization within a specified distance of the $\bar{\Omega}^{\tau}$ threshold are performed (e.g. 5\%), choosing an instance from that host for removal. This lowers the probability that the host will become stressed in the near future. Next, we perform all pending scale up operations, placing new instances first on partially-utilized hosts, and then on underutilized hosts.

Next, we integrate the VM Consolidation operation into the algorithm in order to consolidate load 
onto fewer hosts. The algorithm proceeds similarly to how the operation is describe in Section 5, except that we make use of remaining scale down operations for tasks that have instances on underutilized hosts. First, we calculate a shutdown cost, which is defined as the number of VMs that must be migrated in order to empty the host, assuming that any tasks with pending scale down operations and instances on the candidate host will choose those instances to remove. We then iterate through the remaining scale down operations, choosing instances on underutilized hosts with the lowest shutdown cost for removal. If no task instances are located on an underutilized host, then the task instance on the host with the lowest CPU utilization is chosen. By targeting hosts in this manner, we are intelligently selecting scale down operations such that we remove instances from only a small set of hosts, and increase the likelihood of successfully shutting down an underutilized host with fewer (or zero) migrations required. Finally, if a host has been underutilized for a specified timeout period, we attempt to remove VMs from the host via VM live migration and subsequently shut it down. Based on a evaluation of multiple possible values, we set the timeout to 12 algorithm executions.

\section{Topology-Aware Algorithm}

When placing and dynamically managing applications, consideration should be taken as to where in the topology of the data centre individual components of the application (i.e. VMs) are placed. Individual tasks, and therefore task instances, must communicate with each other in order to complete user requests. We therefore attempt to place all task instances belonging to a single application within the same rack, as described in Section 3.3. The assumption is that such a placement will reduce network latency between communicating task instances, as well as reducing the usage of higher level networking elements. We incorporate the targeting of applications to racks with our integrated algorithm (Section 6) to define a new, topology and application-aware dynamic VM allocation algorithm. In order to enforce the placement of applications within a single rack, both the VM Placement operation and the unified Dynamic Allocation operations must be modified.

\subsection{VM Placement}

The VM Placement operation is modified to make a best effort at placing all VMs of an application within the same rack. It does so in a first fit, greedy manner by first attempting to place the initial request of VMs into each rack in the data centre, executing the placement as soon as a suitable candidate rack is found. If none is found, it then attempts to place the application in two racks, then three, and so on, terminating either when the application is placed or has failed placement on the set of all available hosts. Note that we do not attempt to move existing applications to other racks in order to clear space for the newly arriving application, as the performance overhead of live migrating an entire application is assumed to be unacceptable. Nevertheless, the feasibility of such an approach should be investigated in future work.

The number of applications placed in each rack, and their size, can have an effect on the efficiency of dynamic VM allocation. Too few VMs within a rack can result in a reduction in the ability of the algorithm to effectively consolidate load, and too many results in task instances of an application being spread across multiple racks as applications scale and workload changes. Targeting a specific rack utilization (say, in terms of total CPU usage) is challenging, however, since applications scale up and down with changing workloads, and even individual VMs consume varying amounts of resource. As such, we simply use the number of active (powered on) hosts in a rack as a rough measure of rack utilization, and target a specified number of active hosts $\left(\Upsilon_{h}\right)$. Racks with fewer than $\Upsilon_{h}$ active hosts are sorted in descending order by number of active hosts for placement of a new application. If no placement is found, then placement is attempted using racks with more than $\Upsilon_{h}$ active hosts.

\subsection{Dynamic Allocation}

When performing migrations for VM Relocation (relieving a stressed host), VM Consolidation, or when instantiating a new task instance for a scale up operation, we sort potential target hosts and greedily choose the first successful match. Since hosts are simply sorted based on their utilization, as described in Section 5 and Algorithm 2, this may result in VMs being migrated into a different rack, thus breaking the 
single-rack application placement. In order to correct this situation, we modify the target host sorting. First, we define the majority rack of an application to be the rack which contains more task instances of the application than any other rack. We consider this to be a home rack for the application, and attempt to keep all task instances (VMs) within this rack.

Next, the target host list is divided into two new categories: those within the majority rack $\left(H^{\Re}\right)$ and those outside of it $\left(H^{\Im}\right)$. Each of these categories is then further subdivided into the utilization categories described in Section 5, namely, stressed $\left(H^{!}\right)$, partially utilized $\left(H^{+}\right)$, underutilized $\left(H^{-}\right)$, and empty $\left(H^{\emptyset}\right)$. Finally, we modify the target list construction in Algorithm 2 (line 3) as follows:

$$
\begin{aligned}
\text { targets } \leftarrow & \operatorname{sort}\left(H^{+} \cap H^{\Re}\right) \cdot \operatorname{sort}\left(H^{-} \cap H^{\Re}\right) \cdot \\
& \operatorname{sort}\left(H^{\emptyset} \cap H^{\Re}\right) \cdot \operatorname{sort}\left(H^{+} \cap H^{\Im}\right) \cdot \\
& \operatorname{sort}\left(H^{-} \cap H^{\Im}\right) \cdot \operatorname{sort}\left(H^{\emptyset} \cap H^{\Im}\right)
\end{aligned}
$$

This target list ordering favours hosts within an application's majority rack first, and only resorts to outside hosts when none inside are available. This sorting is performed on a VM-by-VM basis, since each VM may belong to a different application and therefore have a different majority rack. This also leaves room for alternate sort orders to be implemented in order to enforce alternative placement rules (that is, other than single-rack placement).

Despite attempts to contain an application within a single rack, in may be the case that a migration or a scale up operation results in an application becoming spread across more than one rack. We add two measures to combat this: 1) carefully choosing task instances to remove in scale down operations, and 2) correcting placement via migration. Prior to performing VM Consolidation, in the integrated dynamic allocation algorithm described in Section 6, we look for pending scale down operations in which task instances belonging to the task are not located on the applications majority rack. If such operations are found, the instance located on a different rack is chosen for removal, thus completing the scale down operation and resolving the rack placement problem. If there is no such scale down operation pending, and a VM is located outside of its applications majority rack, then we perform a live migration in order to move the VM onto it, if such a target host is available. We refer to this as a placement correction migration.

\section{Evaluation}

In this section, we find suitable tuning parameter values, and evaluate our approach through a series of several experiments. Due to the scale and complexity of the problem domain, experimentation on a real system was not feasible. As such, we evaluate our work using a simulation tool, DCSim [35, 36]. We begin by evaluating our autoscaling algorithm, comparing it against a static allocation for peak application demand. We look at a number of possible tuning parameter values, and select a configuration for use in subsequent experiments. Next, we examine running autoscaling alongside an existing dynamic VM allocation algorithm from our previous work $[10,19]$ (see Section 5), over a number of parameter values. We then perform a similar evaluation of our new, integrated application-aware algorithm, and compare it to running separate autoscaling and dynamic allocation algorithms. Finally, we evaluate our topology-aware modifications to the integrated algorithm.

\subsection{Metrics Collected in the Simulation}

The following metrics are recorded/calculated in the simulation and used to evaluate the presented algorithms.

Active Hosts (Hosts): The average number of hosts in the $O n$ state. The higher the value, the more physical hosts are being used to run the workload. Note that the peak value may be considerably higher.

Average Active Host Utilization (Host Util.): The average CPU utilization of all hosts in the $O n$ state. The higher the value, the more efficiently resources are being used.

Power Consumption (Power): Power consumption is calculated for each host, and the total kilowatthours consumed during the simulation are reported. Power consumption for a host is calculated based on the CPU utilization as calculated in the simulation and the measured power consumption as reported from the SPEC power benchmark [32]. 
Application Size (Size): Application Size is the number of VMs belonging to the application. We report the average size as a percentage of the maximum size defined for each application.

SLA Achievement $(\overline{\boldsymbol{S}})$ : SLA Achievement is the percentage of time in which the SLA conditions are met. See Section 3 for details.

Autoscaling Operations (AS Ops): The number of scale up and scale down operations executed.

Number of Migrations (Migrations): The number of migrations triggered during the simulation. Typically, a lower value is more desirable, as less bandwidth would be used for VM migrations.

Inter-rack Migrations (Inter-rack Migs): The number of migrations in which a VM was moved into a new rack. A lower value is more desirable, as fewer network elements would be involved in migrations.

Placement Correction Migrations (P.C. Migs) The number of migrations performed specifically to correct the rack placement of an application.

Total VM-time (VM-t): The combined total amount of $\mathrm{VM}$ running time. That is, the sum of the running time of every VM that existed at some point in the data centre. In a public IaaS cloud, where clients are charged on a pay-per-use basis (i.e. $x$ dollars per VM per hour), VM-time translates directly into cost. Reported in days.

VM-time Reduction (VM-t Red.): The percentage by which Total VM-time was reduced versus a static allocation of peak resource requirements.

Spread Penalty $(\boldsymbol{S P})$ : The Spread Penalty is a penalty applied when an application(s) in the data centre are placed across more than one rack, thus violating the single-rack application placement objective. It is calculated as the combined total amount of time all applications spent placed on more than one rack, in hours.

\subsection{Experimental Setup}

Our simulated data centre consists of 8 racks of 40 host machines (320 total) based on the HP ProLiant DL160G5, with 2 quad-core $2.5 \mathrm{GHz}$ CPUs and 16 GB of memory. Hosts are modelled to use a workconserving CPU scheduler, as available in major virtualization technologies. As such, CPU shares that are not used by one VM can be used by another. No maximum cap on CPU is set for VMs. When the CPU is at maximum capacity and VMs must compete for resources, VMs are assigned CPU shares in a fairshare manner. Memory is statically allocated and is not overcommitted. We fix the start-up cost of a virtual machine at 30,000 CPU shares; thus, the start-up time is dependent on the CPU of the host that the VM is being placed. For a $2.5 \mathrm{GHz} \mathrm{CPU}$, this would represent 12 seconds.

We model a set of interactive applications running within the data centre, as described in Section 3. Task instances run on VMs with 1 virtual core and $1 \mathrm{~GB}$ of RAM. We defined a set of 4 artificial applications of varying configurations; see Table 2. Application 1 , for example, consists of three tasks, with default size (cores) of 1, 4 and 2, respectively. The service times and visit ratios are used by the closed queuing model representing the application to determine response time. These 4 base applications are then used to create applications during the simulation. The maximum size for each application/task is calculated as its default size is scaled up by a scale factor of 3-6x, chosen randomly. The user think time for each application is set at 4 seconds.

The number of active users changes dynamically for each application and is based on real workload traces. Each application uses a trace built from one of 3 sources: ClarkNet, EPA, and SDSC [22]. We compute a normalized workload level based on request rate, in 100 second intervals, for each trace. These levels are used to define the current number of users of each application. The normalized workloads are

Table 2 Base applications

\begin{tabular}{lllll}
\hline App. & Task & $\begin{array}{l}\text { Service } \\
\text { Time }(\mathrm{sec})\end{array}$ & $\begin{array}{l}\text { Visit } \\
\text { Ratio }\end{array}$ & $\begin{array}{l}\text { Default } \\
\text { Size }\end{array}$ \\
\hline 1 & 1 & 0.005 & 1 & 1 \\
& 2 & 0.02 & 1 & 4 \\
& 3 & 0.01 & 1 & 2 \\
2 & 1 & 0.005 & 1 & 1 \\
& 2 & 0.02 & 1 & 4 \\
3 & 1 & 0.005 & 1 & 1 \\
& 2 & 0.02 & 1 & 4 \\
& 3 & 0.01 & 1 & 2 \\
& 4 & 0.01 & 0.5 & 1 \\
& 5 & 0.02 & 0.5 & 2 \\
& 1 & 0.01 & 1 & 1 \\
\hline
\end{tabular}


scaled such that the peak number of clients receive a response time of 0.9 seconds (just under the defined SLA threshold) when all application tasks have their maximum number of instances. To ensure that applications do not exhibit synchronized behaviour, each applications starts its trace at a randomly selected offset time.

For each experiment, a base load of 10 applications is generated in the first 20 hours (in simulation time) of the experiment, which remain in the data centre for the duration of the experiment. Beginning at the 24 hour mark, additional applications dynamically arrive and depart the system, varying the total number of applications randomly between 10 and 50 every 2 simulated days. These applications have a life span of approximately 2 days, after which they terminate and depart the data centre. Every application is generated with randomly chosen configurations and scale factors. We use 10 different randomly generated application sets to evaluate our work, and all presented results are averaged across experiments using these 10 sets. We run our experiments for 8 simulation days, and we discard data from the first day of simulation to allow the system to stabilize before recording results (resulting in 1 full week of recorded simulation time).

\subsection{Autoscaling}

We compared the autoscaling algorithm (Section 4) against a static allocation. The static allocation creates the maximum number of instances for each application task, places all task instances (VMs) at the start of the experiment, and does not modify the allocation or number of instances further. As such, each application is allocated enough resources to meet peak demand, at all times. In contrast, the autoscaling algorithm attempts to adapt the size of each application to match fluctuating workload demands. Various values for tuning parameters $S^{\text {warn }}$ and $\omega^{\text {safe }}$ were also evaluated (algorithms AS-1 through AS-6). Other tuning parameters, such as $W_{\rho}$, were chosen based on a separate evaluation of potential values. The autoscaling algorithm is executed every 5 minutes, again based on a evaluation of a number of possible frequencies. New task instances from scale up operations are placed in the first available host. During a scale down operation, an arbitrary task instance is chosen, as the autoscaling algorithm is based on potentially client-defined rules and has no knowledge of host state.
Table 3 presents a portion of the results of the autoscaling parameter evaluation. Results for other tested parameter values were omitted as they did not add any significant additional insights. All tested tuning parameter configurations result in a reduction in VM-time and power consumption. As our goal is to preserve SLA performance while reducing costs, we consider AS-6 to be the best configuration of the autoscaling algorithm. It provides a $41 \%$ reduction in total VM-time and a $33 \%$ reduction in power consumption, while maintaining a $95.7 \%$ SLA achievement. As such, we use this configuration in subsequent experiments.

\subsection{Autoscaling with Dynamic VM Allocation}

We now add an existing dynamic VM allocation approach, running alongside our autoscaling algorithm. They run independently, with no knowledge of the actions being performed by the other. The autoscaling algorithm has knowledge only of application metrics, such as response time and VM CPU utilization, and makes decisions accordingly. The dynamic VM allocation algorithm has knowledge of host states, but no knowledge or control over application metrics and operations. New task instances created by the autoscaling algorithm are, however, placed using the VM Placement operation (Section 5). During VM migration, an SLA violation of $10 \%$ of CPU utilization is added to migrating VMs and an additional CPU overhead of $10 \%$ of the migrating VM's CPU utilization is added to both the source and target host [5]. We run a set of 12 different experiments with varying values of $\bar{\Omega}^{\tau}$ and $\underline{\Omega}^{\tau}$. Tuning parameters for the autoscaling algorithm are identical to the values used in algorithm AS-6 in Section 8.3.

Table 4 presents the results of our experiments. Compared to the autoscaling algorithm alone (Table 3), we see a $25-33 \%$ savings in power consumption. This, however, comes at the expense of SLAs. While lower $\bar{\Omega}^{\tau}$ values (upper limit on percent of CPU utilization used to determine stressed hosts) lead to better SLA achievement, they also lead to a significant increase in the number of migrations performed. In addition, there is an increase seen in the number of autoscaling operations, which indicates that consolidation is affecting the behaviour of the autoscaling algorithm. Decreasing $\underline{\Omega}^{\tau}$ trades increased power consumption for improved SLA and 
Table 3 Autoscaling algorithm (AS)

\begin{tabular}{llllllllll}
\hline Alg & $S^{\text {warn }}$ & $\omega^{\text {safe }}$ & Hosts & Power & $\bar{S}$ & Size & AS Ops & VM-t & VM-t Red. \\
\hline Static & N/A & N/A & 157.47 & $4735 \mathrm{kWh}$ & $100.0 \%$ & $100 \%$ & N/A & 5605 days & N/A \\
\hline AS-1 & 0.9 & 0.5 & 73.5 & $2502 \mathrm{kWh}$ & $87.6 \%$ & $46 \%$ & 5175 & 2523 days & $55 \%$ \\
AS-2 & 0.9 & 0.3 & 85.6 & $2845 \mathrm{kWh}$ & $89.5 \%$ & $51 \%$ & 3878 & 2881 days & $49 \%$ \\
AS-3 & 0.8 & 0.3 & 87.1 & $2885 \mathrm{kWh}$ & $90.8 \%$ & $52 \%$ & 4066 & 2931 days & $48 \%$ \\
AS-4 & 0.6 & 0.3 & 90.0 & $2964 \mathrm{kWh}$ & $92.8 \%$ & $54 \%$ & 4479 & 3040 days & $46 \%$ \\
AS-5 & 0.3 & 0.5 & 85 & $2826 \mathrm{kWh}$ & $94.7 \%$ & $53 \%$ & 7171 & 2918 days & $48 \%$ \\
AS-6 & $\mathbf{0 . 3}$ & $\mathbf{0 . 3}$ & $\mathbf{9 7 . 6}$ & $\mathbf{3 1 6 8} \mathbf{k W h}$ & $\mathbf{9 5 . 7 \%}$ & $\mathbf{5 9 \%}$ & $\mathbf{5 4 9 5}$ & $\mathbf{3 3 0 1}$ days & $\mathbf{4 1 \%}$ \\
\hline
\end{tabular}

migration count. Based on the results, we argue that using $\bar{\Omega}^{\tau}=80 \%$ and $\underline{\Omega}^{\tau}=40 \%$ is the best choice of configuration for the separate dynamic VM allocation algorithm, as it provides significant power savings while maintaining good SLA achievement and requiring significantly fewer migrations than with a further reduced $\bar{\Omega}^{\tau}$. This, of course, is valid only for our particular experimental configuration, but serves to show both the importance of the parameter values as well as the control they provide over the algorithm. In the future, these values could be determined automatically via a machine learning algorithm. We use this configuration for further comparison in Section 8.7.

\subsection{Integrated Algorithm}

We now evaluate our newly developed integrated autoscaling and dynamic VM allocation algorithm. Once again, we run a set of 12 different experiments with varying values of $\bar{\Omega}^{\tau}$ and $\underline{\Omega}^{\tau}$. Autoscaling decisions are made in the evaluateScaling() method (see Algorithm 3) using the same autoscaling tuning parameter values as algorithm AS-6 in Section 8.3, to provide a fair comparison.

Table 5 presents the results of our experiments with the integrated algorithm. Compared with separate autoscaling and dynamic VM allocation algorithms, we can see a notable improvement in SLA achievement, reaching nearly that of autoscaling alone. Furthermore, we note a significant decrease in the number of migrations, falling in line with our goals for the development of the algorithm, as well as fewer autoscaling operations. Again, we argue that using $\bar{\Omega}^{\tau}=80 \%$ and $\underline{\Omega}^{\tau}=40 \%$ is the best choice of configuration for the integrated algorithm, as it provides significant power savings, sacrifices very little in terms of SLA achievement, and exhibits one of the lowest migration counts. We use this configuration for further comparison in Section 8.7.

\subsection{Topology-aware Algorithm}

Finally, we examine the performance of the complete, topology- and application-aware algorithm. This algorithm is similar to the integrated algorithm, modified

Table 4 Separate autoscaling and dynamic virtual machine allocation (AS+DVM)

\begin{tabular}{|c|c|c|c|c|c|c|c|c|}
\hline $\bar{\Omega}^{\tau}$ & $\underline{\Omega}^{\tau}$ & Hosts & Host Util. & Power & $\bar{S}$ & Migrations & AS Ops & VM-t \\
\hline 90 & 60 & 57.7 & $78.6 \%$ & $2116 \mathrm{kWh}$ & $89.0 \%$ & 9653 & 7144 & 3824 days \\
\hline 90 & 40 & 58.3 & $77.9 \%$ & $2132 \mathrm{kWh}$ & $88.9 \%$ & 8350 & 7129 & 3818 days \\
\hline 85 & 60 & 59.5 & $77.0 \%$ & $2172 \mathrm{kWh}$ & $90.2 \%$ & 16481 & 7043 & 3772 days \\
\hline 85 & 40 & 59.9 & $76.3 \%$ & $2181 \mathrm{kWh}$ & $90.2 \%$ & 14688 & 7002 & 3758 dayss \\
\hline 80 & 60 & 61.6 & $74.8 \%$ & $2233 \mathrm{kWh}$ & $91.5 \%$ & 23734 & 6886 & 3698 days \\
\hline 80 & 40 & 62.6 & $73.8 \%$ & $2260 \mathrm{kWh}$ & $91.6 \%$ & 21337 & 6891.7 & 3703 days \\
\hline 75 & 60 & 64.9 & $72.0 \%$ & $2326 \mathrm{kWh}$ & $92.7 \%$ & 31297 & 6792 & 3657 days \\
\hline 75 & 40 & 65.8 & $70.9 \%$ & $2349 \mathrm{kWh}$ & $92.6 \%$ & 27886 & 6779 & 3648 days \\
\hline
\end{tabular}


Table 5 Integrated algorithm (INT)

\begin{tabular}{|c|c|c|c|c|c|c|c|c|}
\hline $\bar{\Omega}^{\tau}$ & $\underline{\Omega}^{\tau}$ & Hosts & Host Util. & Power & $\bar{S}$ & Migrations & AS Ops & VM-t \\
\hline 90 & 60 & 57.8 & $78.6 \%$ & $2122 \mathrm{kWh}$ & $88.8 \%$ & 6612 & 7241 & 3855 days \\
\hline 90 & 40 & 70.2 & $66.9 \%$ & $2458 \mathrm{kWh}$ & $93.6 \%$ & 3471 & 6646 & 3600 days \\
\hline 85 & 60 & 58.3 & $78.0 \%$ & $2136 \mathrm{kWh}$ & $89.6 \%$ & 6652 & 7136 & 3805 days \\
\hline 85 & 40 & 70.9 & $66.7 \%$ & $2480 \mathrm{kWh}$ & $93.9 \%$ & 3924 & 6634 & 3604 days \\
\hline 80 & 60 & 59.5 & $77.1 \%$ & $2172 \mathrm{kWh}$ & $90.7 \%$ & 6954 & 7093 & 3760 days \\
\hline 80 & 40 & 70.5 & $66.6 \%$ & $2469 \mathrm{kWh}$ & $94.1 \%$ & 4346 & 6573 & 3573 days \\
\hline 75 & 60 & 61.0 & $75.8 \%$ & $2220 \mathrm{kWh}$ & $91.7 \%$ & 7304 & 7016 & 3734 days \\
\hline 75 & 40 & 71.0 & $66.1 \%$ & $2483 \mathrm{kWh}$ & $94.2 \%$ & 4815 & 6520 & 3563 days \\
\hline
\end{tabular}

to attempt to keep each application within a single rack (see Section 7). First, we evaluate a range of potential values for the rack utilization target parameter $\left(\Upsilon_{h}\right)$. Table 6 presents the results of these experiments. We can see that power consumption decreases slightly with higher rack utilization targets, but not by a large amount. SLA achievement and migration counts are best in the mid-range values, and interrack migrations, placement correction migrations, and spread penalty all increase with rack utilization target. We use $\Upsilon_{h}=0.5$ for our final evaluation, as it provides the best SLA, is close to the lowest migration count, and does not exhibit the increase in inter-rack migrations and spread penalty seen in higher values.

In Table 7 we compare the results of the integrated, application-aware algorithm (INT) with the topologyaware and application-aware algorithm (INT+TOPO). The topology-aware algorithm sacrifices a small amount of SLA achievement (92.9\% versus $94.1 \%$ ), and exhibits an increase in total migrations. On the other hand, topology-aware significantly reduces the number of inter-rack migrations and the application spread penalty, thus demonstrating that it achieves the goal of trying to contain each application within a single rack.

\subsection{Discussion}

Table 8 presents a comparison between the evaluated algorithms over four different metrics. STATIC refers to a static allocation of peak resource requirements; $A S$ is the autoscaling algorithm running alone; $A S+D V M$ is the autoscaling and dynamic VM allocation algorithms running separately; INT is the integrated application-aware algorithm; and INT+TOPO is the topology- and application-aware algorithm. Note that we compare SLA Violation, which is the inverse of SLA achievement, and as such lower values are better.

The static allocation (STATIC) naturally involved no SLA violations or migrations, but compared to all other algorithms, has the highest VM-time and power

Table 6 Topology-aware (INT+TOPO) rack target $\left(\Upsilon_{h}\right)$

\begin{tabular}{|c|c|c|c|c|c|c|c|}
\hline$\Upsilon_{h}$ & Power & $\bar{S}$ & Migrations & Inter-rack Migs & P.C. Migs & VM-t & SP \\
\hline 0.1 & $2465 \mathrm{kWh}$ & $92.0 \%$ & 7628 & 3.2 & 3.2 & 3718 & 0 \\
\hline 0.2 & $2466 \mathrm{kWh}$ & $92.3 \%$ & 6620 & 9 & 8.7 & 3685 & 1.3 \\
\hline 0.3 & $2460 \mathrm{kWh}$ & $92.6 \%$ & 6310 & 17.4 & 17.4 & 3662 & 0.1 \\
\hline 0.4 & $2469 \mathrm{kWh}$ & $92.7 \%$ & 5741 & 60.8 & 59.4 & 3659 & 5.9 \\
\hline 0.5 & $2458 \mathrm{kWh}$ & $\mathbf{9 2 . 9} \%$ & 5484 & 149.7 & 144.1 & 3659 & 22.3 \\
\hline 0.6 & $2442 \mathrm{kWh}$ & $92.8 \%$ & 5386 & 286.9 & 278 & 3658 & 33.7 \\
\hline 0.7 & $2435 \mathrm{kWh}$ & $92.7 \%$ & 5392 & 572.3 & 549.1 & 3675 & 79.6 \\
\hline 0.8 & $2411 \mathrm{kWh}$ & $92.4 \%$ & 5515 & 1082.7 & 1000.6 & 3696 & 182.6 \\
\hline 0.9 & $2387 \mathrm{kWh}$ & $92.3 \%$ & 5523 & 1600.4 & 1422.6 & 3696 & 280.5 \\
\hline 1.0 & $2348 \mathrm{kWh}$ & $91.8 \%$ & 5714 & 2397.7 & 2032.4 & 3698 & 486.2 \\
\hline
\end{tabular}


Table 7 Topology-aware vs topology-unaware

\begin{tabular}{lllllllcc}
\hline Alg. & Power & $\bar{S}$ & Migs & Inter-rack Migs & P.C. Migs & AS Ops & VM-t & SP \\
\hline INT & $2469 \mathrm{kWh}$ & $94.1 \%$ & 4346 & 2601 & N/A & 6573 & 3573 days & 3481.7 \\
INT+TOPO & $2458 \mathrm{kWh}$ & $92.9 \%$ & 5484 & 149.7 & 144 & 6798 & 3659 days & 22.3 \\
\hline
\end{tabular}

consumption. This represents higher costs for both the cloud provider (power costs) and cloud client (VM rental costs). The autoscaling algorithm $(A S)$ allows some SLA violations, but significantly reduces power consumption and VM-time compared to the static allocation. Further improvements in power consumption can be made through the use of dynamic VM allocation in the remaining algorithms. The $A S+D V M$ algorithm results in the most power savings, but at the expense of the worst SLA violations and a very large migration count. The INT algorithm corrects this problem, offering similar power savings ( $22 \%$ better than $A S$ ) while drastically reducing the required number of migrations. Finally, the INT+TOPO algorithm loses a bit in SLA violations, power consumption and migrations, but successfully keeps each application within a single rack, which has additional benefits not directly reflected in this evaluation (see Section 7). Total VM-time for all experiments featuring dynamic VM allocation was slightly higher than for $A S$, indicating that dynamic VM allocation caused additional scale up operations to be triggered due to $\mathrm{CPU}$ contention.

We draw the following conclusions from this work:

- Autoscaling is important in reducing the time VMs consume and the number of SLA violations; it can also help to reduce power consumption.

- Using dynamic VM allocation when combined with autoscaling can further reduce power consumption; something clearly of interest to cloud providers. This comes, however, at costs for more migrations and increased SLA violations.
- The integrated autoscaling and dynamic VM allocation algorithm that is application aware introduced in this paper overcomes the problems of using both autoscaling and dynamic VM allocation separately. This would seem to be a very good strategy for both cloud providers and for cloud clients.

- Augmenting the integrated algorithm with topology-aware considerations does cluster VMs belonging to the same application together in racks at some cost to SLA violations. The real test for the value of this algorithm would be in network usage and latency. This requires an extension to the current simulator.

\section{Conclusions and Future Work}

Both cloud providers and clients must strive to make efficient use of their resources in order to reduce their infrastructure requirements and costs. Cloud providers must consolidate load onto as few physical machines as possible in order to reduce power consumption and/or serve more clients with less hardware, while still providing the resources that their clients require. Cloud clients should provision only the resources they require to meet their current demand and objectives. To this end, application autoscaling dynamically adds and removes resources (VMs) to and from an application as demand changes. We introduced a rule-based heuristic autoscaling algorithm to address cloud client needs, and ran it alongside a dynamic VM allocation
Table 8 Overall algorithm comparison

\begin{tabular}{lllll}
\hline Alg. & SLA Viol.(\%) & \# Migs. & VM-time (days) & Power (kwh) \\
\hline STATIC & 0 & 0 & 5605 & 4735 \\
AS & 4.3 & 0 & 3301 & 3168 \\
AS+DVM & 8.4 & 21337 & 3703 & 2260 \\
INT & 5.9 & 4346 & 3573 & 2469 \\
INT+TOPO & 7.1 & 5484 & 3659 & 2458 \\
\hline
\end{tabular}


algorithm from our previous work [10, 19] to perform consolidation. While power consumption was reduced, it was at the cost of SLA achievement and a large number of VM live migrations. We developed a new, integrated application-aware algorithm which leverages some control over autoscaling operations to assist dynamic VM allocation. Through this approach, we were able to improve SLA performance and greatly reduce the number of migrations required. Our final objective was to consider the data centre network topology when placing applications. To this end, we introduced a topology-aware aspect to the integrated algorithm, which attempts to place each application entirely within a single rack. The modifications were successful in achieving this goal, although it was at a slight cost in SLA and additonal migrations. That said, VM-to-VM communication and migrations were almost entirely contained within a single rack, reducing the load on upper level networking elements.

Future work includes investigating methods for reducing the effect of dynamic $\mathrm{VM}$ allocation on total VM-time. A more intelligent autoscaling algorithm could also be investigated, with the potential for each application to make use of a different, custom algorithm. A number of tuning parameters influence the performance of the autoscaling and dynamic VM allocation algorithms. Rather than determining values for these parameters experimentally, a machine learning approach could be developed to automatically discover the best values for each environment. More complex placement constraints for application tasks and task instances, such as specific placements for fault tolerance, could also be considered. Finally, we have previously developed a distributed algorithm for dynamic VM allocation [37], which could be extended to handle both autoscaling and dynamic VM allocation.

Open Access This article is distributed under the terms of the Creative Commons Attribution 4.0 International License (http:// creativecommons.org/licenses/by/4.0/), which permits unrestricted use, distribution, and reproduction in any medium, provided you give appropriate credit to the original author(s) and the source, provide a link to the Creative Commons license, and indicate if changes were made.

\section{References}

1. Amazon: Amazon EC2 Auto Scale. http://aws.amazon. com/autoscaling/ (2013)
2. Ballani, H., Costa, P., Karagiannis, T., Rowstron, A.: Towards predictable datacenter networks. ACM SIGCOMM Comput. Commun. Rev. 41(4), 242-253 (2011)

3. Barker, S.K., Shenoy, P.: Empirical evaluation of latencysensitive application performance in the cloud. In: Proceedings of the first annual ACM SIGMM conference on multimedia systems, pp. 35-46. ACM (2010). doi:10.1145/1730836.1730842

4. Barroso, L., Holzle, U.: The case for energy-proportional computing. Computer 40(12), 33-37 (2007)

5. Beloglazov, A., Buyya, R.: Optimal online deterministic algorithms and adaptive heuristics for energy and performance efficient dynamic consolidation of virtual machines in cloud data centers. Concurr. Computat. Pract. Exper., 1-24 (2011)

6. Bobroff, N., Kochut, A., Beaty, K.: Dynamic placement of virtual machines for managing sla violations. In: Proceedings of the 2007 IEEE/IFIP International Symposium on IM, pp. 119-128 (2007). doi:10.1109/INM.2007.374776

7. Cardosa, M., Korupolu, M.R., Singh, A.: Shares and utilities based power consolidation in virtualized server environments. In: Proceedings of the 2009 IEEE/IFIP international symposium IM, pp. 299-302 (2009)

8. Dias, D.S., Costa, L.H.M.: Online traffic-aware virtual machine placement in data center networks. In: Global information infrastructure and networking symposium (GIIS), pp. 1-8. IEEE (2012)

9. Ferretti, S., Ghini, V., Panzieri, F., Pellegrini, M., Turrini, E.: Qos-aware clouds. In: 2010 IEEE 3rd international conference on cloud computing (CLOUD), pp. 321-328. IEEE (2010)

10. Foster, G., Keller, G., Tighe, M., Lutfiyya, H., Bauer, M.: The right tool for the job: switching data centre management strategies at runtime. In: 2013 IFIP/IEEE international symposium on integrated network management (IM), pp. 151-159 (2013)

11. Galante, G., de-Bona, L.C.E.: A survey on cloud computing elasticity. In: IEEE/ACM fifth international conference on utility and cloud computing, pp. 263-270 (2012). doi:10.1109/UCC.2012.30

12. Ghanbari, H., Simmons, B., Litoiu, M., Barna, C., Iszlai, G.: Optimal autoscaling in a iaas cloud. In: Proceedings of the 9th international conference on autonomic computing, pp. 173-178. ACM (2012)

13. Ghanbari, H., Simmons, B., Litoiu, M., Iszlai, G.: Exploring alternative approaches to implement an elasticity policy. In: 2011 IEEE international conference on cloud computing (CLOUD), pp. 716-723. IEEE (2011)

14. Gmach, D., Rolia, J., Cherkasova, L., Belrose, G., Turicchi, T., Kemper, A.: An integrated approach to resource pool management: policies, efficiency and quality metrics. In: 38th Annual IEEE/IFIP international conference on dependable systems and networks (DSN), pp. 326-335 (2008). doi:10.1109/DSN.2008.4630101

15. Guo, C., Lu, G., Wang, H.J., Yang, S., Kong, C., Sun, P., Wu, W., Zhang, Y.: Secondnet: a data center network virtualization architecture with bandwidth guarantees. In: Proceedings of the 6th international conference, p. 15. ACM (2010)

16. Gupta, A., Milojicic, D., Kalé, L.V.: Optimizing Vm placement for Hpc in the cloud. In: Proceedings of the 2012 
workshop on cloud services, federation, and the 8th open cirrus summit, pp. 1-6. ACM (2012)

17. Hyser, C., Mckee, B., Gardner, R., Watson, B.J.: Autonomic virtual machine placement in the data center. Tech. Rep. HPL-2007-189 HP Laboratories (2007)

18. Jung, G., Joshi, K.R., Hiltunen, M.A., Schlichting, R.D., $\mathrm{Pu}, \mathrm{C} .: \mathrm{A}$ cost-sensitive adaptation engine for server consolidation of multitier applications. In: Middleware 2009, pp. 163-183. Springer (2009)

19. Keller, G., Tighe, M., Lutfiyya, H., Bauer, M.: An analysis of first fit heuristics for the virtual machine relocation problem. In: Proceedings of the 6th international DMTF academic alliance workshop on SVM, pp. 406-413 (2012)

20. Kephart, J., Chess, D.: The vision of autonomic computing. IEEE Comput. 36(1), 41-50 (2003)

21. Khanna, G., Beaty, K., Kar, G., Kochut, A.: Application performance management in virtualized server environments. In: NOMS Proceedings, 2006 IEEE/IFIP, pp. 373381 (2006). doi:10.1109/NOMS.2006.1687567

22. Lawrence Berkeley National Laboratory: The internet traffic archive. http://ita.ee.lbl.gov/ (2013)

23. Lorido-Botran, T., Miguel-Alonso, J., Lozano, J.A.: A review of auto-scaling techniques for elastic applications in cloud environments. J. Grid Comput. 12(4), 559-592 (2014). doi:10.1007/s10723-014-9314-7

24. Mann, V., Gupta, A., Dutta, P., Vishnoi, A., Bhattacharya, P., Poddar, R., Iyer, A.: Remedy: network-aware steady state Vm management for data centers. In: NETWORKING 2012, pp. 190-204. Springer (2012)

25. Maurer, M., Breskovic, I., Emeakaroha, V., Brandic, I.: Revealing the MAPE loop for the autonomic management of cloud infrastructures. In: IEEE symposium on computers and communications (ISCC), pp. 147-152 (2011). doi:10.1109/ISCC.2011.5984008

26. Naskos, A., Gounaris, A., Sioutas, S.: A review of autoscaling techniques for elastic applications in cloud environments. LNCS 9511 ALGOCLOUD 2015 pp. 151-167 (2016). doi:10.1007/978-3-319-29919-8 12

27. Petrucci, V., Carrera, E.V., Loques, O., Leite, J.C., Mossé, D.: Optimized management of power and performance for virtualized heterogeneous server clusters. In: 2011 11Th IEEE/ACM international symposium on cluster, cloud and grid computing (CCGrid), pp. 23-32. IEEE (2011)

28. Reiser, M., Lavenberg, S.S.: Mean-value analysis of closed multichain queuing networks. J. ACM (JACM) 27(2), 313322 (1980). doi:10.1145/322186.322195

29. Shen, Z., Subbiah, S., Gu, X., Wilkes, J.: Cloudscale: elastic resource scaling for multi-tenant cloud systems. In: Proceedings of the 2 nd ACM symposium on cloud computing, p. 5. ACM (2011)

30. Singh, S., Chana, I.: A survey on resource scheduling in cloud computing: Issues and challenges. J. Grid Comput. 14(2), 217-264 (2016). doi:10.1007/s10723-015-9359-2

31. Speitkamp, B., Bichler, M.: A mathematical programming approach for server consolidation problems in virtualized data centers. IEEE TSC 3(4), 266-278 (2010). doi:10.1109/TSC.2010.25
32. Standard Performance Evaluation Corporation: Specpower_ ssj2008 benchmark. http://www.spec.org/power_ssj2008/ (2013)

33. Stillwell, M., Schanzenbach, D., Vivien, F., Casanova, H.: Resource allocation algorithms for virtualized service hosting platforms. J. Parallel Distrib. Comput. 70(9), 962-974 (2010). doi:10.1016/j.jpdc.2010.05.006

34. Tighe, M., Bauer, M.: Integrating cloud application autoscaling with dynamic VM allocation. In: IEEE/ IFIP network operations and management symposium (NOMS), pp. 1-9. IEEE (2014)

35. Tighe, M., Keller, G.: DCSim on GitHub. https://github. com/digs-uwo/dcsim (2013)

36. Tighe, M., Keller, G., Bauer, M., Lutfiyya, H.: Towards an improved data centre simulation with DCSim. In: Proceedings of the 7th international DMTF academic alliance workshop on SVM, pp. 364-372 (2013)

37. Tighe, M., Keller, G., Lutfiyya, H., Bauer, M.: A distributed approach to dynamic VM management. In: 2013 9th international conference on network and service management (CNSM). IEEE (2013)

38. Verma, A., Ahuja, P., Neogi, A.: pMapper: power and migration cost aware application placement in virtualized systems. In: Proceedings of the 9th ACM/IFIP/USENIX international conference on middleware, pp. 243-264 (2008)

39. Wang, G., $\mathrm{Ng}$, T.S.E.: The impact of virtualization on network performance of Amazon EC2 data center. In: Proceedings IEEE INFOCOM, pp. 469-482 (2010). doi:10.1109/INFCOM.2010.5461931

40. Wood, T., Shenoy, P., Venkataramani, A., Yousif, M.: Black-box and gray-box strategies for virtual machine migration. In: Proceedings of the 4th symposium on NSDI, pp. 229-242, Cambridge, MA, USA (2007)

41. Wuhib, F., Stadler, R., Spreitzer, M.: Gossip-based resource management for cloud environments. In: 2010 international conference on network and service management (CNSM), pp. 1-8. IEEE (2010)

42. Yanggratoke, R., Wuhib, F., Stadler, R.: Gossip-based resource allocation for green computing in large clouds. In: 20117 th international conference on network and service management (CNSM), pp. 1-9. IEEE (2011)

43. Yapicioglu, T., Oktug, S.: A traffic-aware virtual machine placement method for cloud data centers. In: Proceedings of the 2013 IEEE/ACM 6th international conference on utility and cloud computing, pp. 299-301. Computer Society (2013)

44. Zhani, M.F., Zhang, Q., Simon, G., Boutaba, R.: VDC planner: dynamic migration-aware virtual data center embedding for clouds. In: IEEE/IFIP integrated network management symposium (IM), pp. 18-25. IEEE (2013)

45. Zhu, Q., Zhu, J., Agrawal, G.: Power-aware consolidation of scientific workflows in virtualized environments. In: ACM/IEEE international conference for high performance computing, networking, storage and analysis, pp. 1-12 (2010) 